\title{
PRODUÇÃO DE BIOSSURFACTANTE POR Aspergillus fumigatus UTILIZANDO RESÍDUOS AGROINDUSTRIAIS COMO SUBSTRATO
}

\author{
Gabriel Luis Castiglioni, Telma Elita Bertolin e Jorge Alberto Vieira Costa* \\ Escola de Química e Alimentos, Universidade Federal do Rio Grande, CP 474, 96201-900 Rio Grande - RS, Brasil
}

Recebido em 16/12/07; aceito em 28/8/08; publicado na web em 12/2/09

\begin{abstract}
SOLID-STATE BIOSURFACTANT PRODUCTION BY Aspergillus fumigatus USING AGRICULTURAL RESIDUES AS SUBSTRATE. The objective of this study was to investigate biosurfactant production in solid state by Aspergillus fumigatus in fixedbed column bioreactors using substrate based on agricultural residues. Without a supplementary carbon source the highest emulsifying activity (EA) was 11.17 emulsifying units (EU) $\mathrm{g}^{-1}$ of substrate at an aeration rate of $148 \mathrm{~mL} \mathrm{~h}^{-1} \mathrm{~g}^{-1}$ but in the presence of diesel oil the highest EA value was $9.99 \mathrm{EU} \mathrm{g}^{-1}$ at an aeration rate of $119 \mathrm{~mL} \mathrm{~h}^{-1} \mathrm{~g}^{-1}$ of substrate while supplementation with soya oil resulted in only $8.47 \mathrm{EU} \mathrm{g}^{-1}$ of substrate at an aeration rate of $119 \mathrm{~mL} \mathrm{~h}^{-1} \mathrm{~g}^{-1}$.
\end{abstract}

Keywords: filamentous fungi; biosurfactant; solid-state fermentation.

\section{INTRODUÇÃO}

Mudanças realizadas nos processos de fermentação em estado sólido (FES) têm mostrado vantagens quanto ao rendimento e potencial de recuperação de produtos, quando comparados aos processos submersos. ${ }^{1}$ Devido à capacidade de crescimento em baixos níveis de água nos sistemas de FES, os fungos filamentosos têm recebido maior atenção nas pesquisas. ${ }^{2}$ Culturas de Rhizopus, Tríchoderma, Penicilium e Aspergillus são exemplos de alguns fungos utilizados para obtenção de vários bioprodutos por FES. ${ }^{3}$

A síntese microbiana dos biossurfactantes ocorre especialmente durante o crescimento em substratos imiscíveis em água. Devido à baixa toxicidade e alta biodegradabilidade, estas moléculas podem ser usadas amplamente no processamento de alimentos (emulsificantes e umectantes), na indústria de cosméticos, fármacos, detergentes, entre outros. Além disso, são ecologicamente seguros e podem ser aplicados nos processos de biorremediação e tratamento de efluentes. ${ }^{4}$

O potencial de aplicação de compostos de superfície ativa, como é caso dos biossurfactantes, é baseado nas propriedades de emulsificação, umedecimento, solubilização, de-mulsificação, separação, inibição de corrosão, redução de viscosidade de líquidos, entre outros. ${ }^{56}$

Os biossurfactantes apresentam a capacidade de emulsificar misturas de hidrocarbonetos em água. Isto pode ser demonstrado através do aumento significativo da degradação dos hidrocarbonetos presentes no meio, o que torna sua utilização em processos de biorremediação de solos e mananciais contaminados muito freqüiente. ${ }^{7}$ Esse processo dinâmico é baseado na habilidade dos biossurfactantes reduzirem a tensão superficial pelo remanejamento molecular, através do acúmulo de compostos insolúveis em superfícies, influenciando as ligações de hidrogênio e interações hidrofílicas-hidrofóbicas. Isto faz com que a área superficial aumente, proporcionando uma maior biodisponibilidade e conseqüente aumento da biodegradabilidade. ${ }^{8}$

As modificações das condições fisiológicas e da composição do meio de cultivo são algumas alternativas que vêm sendo estudadas para o aumento da produtividade dos biossurfactantes. ${ }^{9}$ A produção de biossurfactante dispensa o uso de substratos de alto custo, podendo ser

\footnotetext{
*e-mail: dqmjorge@furg.br
}

utilizados resíduos agroindustriais, o que economicamente viabiliza o processo, uma vez que o meio de cultivo representa aproximadamente $50 \%$ do custo final do produto. ${ }^{10}$

Um dos grandes mercados para os biossurfactantes é a indústria petrolífera, onde são usados na produção do petróleo e na incorporação de óleos formulados. Durante a última década, a demanda de surfactantes aumentou em aproximadamente $300 \%$ na indústria química dos EUA. ${ }^{11}$ Sua produção mundial excede 3 milhões de t por ano, o que corresponde 4 bilhões de dólares. ${ }^{12}$ Melhorar a recuperação de óleo também representa um grande futuro para o mercado de biossurfactantes. ${ }^{13}$

Tendo em vista as observações mencionadas anteriormente, o presente trabalho teve por objetivo produzir biossurfactante de Aspergillus fumigatus por fermentação em estado sólido, variando-se a vazão de ar e a fonte adicional de carbono no meio de cultivo.

\section{PARTE EXPERIMENTAL}

\section{Microrganismo}

O microrganismo utilizado nos experimentos foi a cepa do fungo filamentoso Aspergillus fumigatus, isolado de um local contaminado por hidrocarbonetos situado no estado de São Paulo (Brasil). Esta cepa foi cedida pelo Laboratório de Microbiologia de Alimentos, da Faculdade de Engenharia de Alimentos da Universidade Estadual de Campinas (FEA/UNICAMP), mantida em tubos de ensaio, com agar batata-dextrose (PDA) adicionados de $1 \%(\mathrm{v} / \mathrm{v})$ de glicerina a $4{ }^{\circ} \mathrm{C}$.

\section{Fermentação em estado sólido (FES)}

Os cultivos foram conduzidos no sistema descrito por Hasan et $a l .{ }^{14}$ conforme apresentado na Figura 1. As fermentações realizadas em biorreatores de coluna encamisada (7), com dimensões internas de $50 \mathrm{~mm}$ de diâmetro e $250 \mathrm{~mm}$ de altura, foram mantidas em banhomaria termostatizado (1) durante $144 \mathrm{~h}$. $\mathrm{O}$ ar fornecido por bombas de diafragma (2) foi filtrado (3), umidificado (5) e transferido para o eliminador de gotas (6), com o objetivo de manter a umidade do ar constante. A injeção de ar de cada experimento foi controlada através de rotâmetros (4) instalados na base de cada biorreator. 


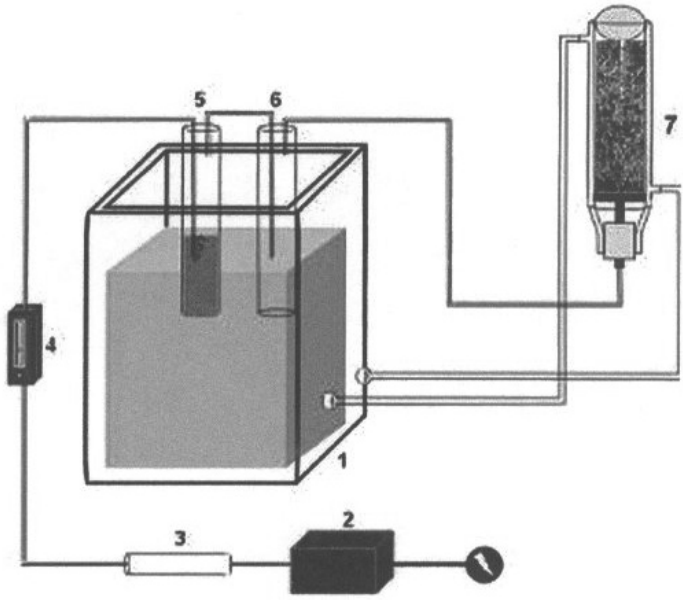

Figura 1. Representação esquemática da fermentação em estado sólido realizada em biorreatores de coluna

O meio de cultivo foi composto por $250 \mathrm{~g}$ de casca e farelo de arroz (15:85). O farelo foi moído em moinho de facas, utilizando malha de $1 \mathrm{~mm}$. Após a moagem, o farelo foi peneirado e as partículas com diâmetro entre 0,42 e $0,50 \mathrm{~mm}$ foram utilizadas nos experimentos. Além da casca e do farelo de arroz, também fez parte do meio fermentativo $90 \mathrm{~mL}$ de solução nutriente composta por $(\mathrm{g}$ $\left.\mathrm{L}^{-1}\right)$ : MgSO4.7H $\mathrm{O}(0,5), \mathrm{NaNO}_{3}(3,0), \mathrm{KH}_{2} \mathrm{PO}_{4}(1,0)$, extrato de levedura $(1,0)$ e peptona $(0,3)$.

Óleo de soja e óleo diesel foram adicionados na proporção de $1 \%$ $\left(\mathrm{w} \mathrm{W}^{-1}\right)$, como fonte adicional de carbono em experimentos separados, para posterior comparação com experimentos na sua ausência. Além de cultivos sem alimentação de ar, foram avaliadas as aerações 40, 60, 100,120 e $200 \mathrm{~mL}_{\mathrm{ar}} \mathrm{g}_{\text {meio }}{ }^{-1} \mathrm{~h}^{-1}$. As condições físico-químicas utilizadas nos experimentos foram $30^{\circ} \mathrm{C}$, concentração inicial de esporos $4.10^{6}$ esporos $\mathrm{g}_{\text {meio }}{ }^{-1}, \mathrm{pH} 4,5$ e umidade $50 \%$.

\section{Extração do biossurfactante}

A extração do biossurfactante do meio fermentado foi realizada com água destilada a $90{ }^{\circ} \mathrm{C}$ na proporção $1: 5$ (uma parte de soluto para cinco partes de solvente). Após a adição do solvente, a amostra foi submetida à agitação em shaker Certomat, BS-1 a 160 rpm e 50 ${ }^{\circ} \mathrm{C}$ por $30 \mathrm{~min}$. A seguir, as amostras foram filtradas a vácuo em funil de Büchner, obtendo os extratos para análise.

\section{Determinações analíticas}

As amostras foram coletadas em intervalos de $24 \mathrm{~h}$ e todas as análises realizadas em triplicata. A umidade foi determinada segundo a metodologia da AOAC, ${ }^{15}$ para posterior conversão dos resultados para base seca.

A atividade emulsificante (AE) foi determinada utilizando a metodologia descrita por Johnson et al. ${ }^{16} \mathrm{O}$ filtrado proveniente da extração do biossurfactante juntamente com água destilada e óleo de soja foi agitado durante $1 \mathrm{~min}$, deixado em repouso por $60 \mathrm{~min}$ e posteriormente submetido à leitura em espectrofotômetro. A atividade emulsificante foi expressa em unidades emulsificantes $\left(\mathrm{UE} \mathrm{g}^{-1}\right)$, definida como a massa de biossurfactante necessária para aumentar a absorbância (Abs) em 1,0 unidade à $610 \mathrm{~nm}$, quando comparada com o branco (Equação 1).

$$
\mathrm{AE}=\frac{\text { Abs.D }}{[\mathrm{m} \cdot(1-\mathrm{U})]}
$$

onde: $\mathrm{Abs}=$ absorbância da suspensão do óleo em água; $\mathrm{D}=$ diluição da amostra em água; $\mathrm{m}=$ massa da amostra úmida (g); $\mathrm{U}=$ umidade do meio fermentado.

\section{Tratamento dos resultados}

Os resultados foram avaliados primeiramente através de análise estatística utilizando o software R, versão 2.0.0 (Core Team), seguida das análises de variância uni e multivariada. Nesta última, foi realizada análise de cluster utilizando o método de Manhattan, considerando a distância entre os resultados de AE mais próximos como a distância entre os agrupamentos.

Em cada condição de aeração foram coletados resultados ótimos de $\mathrm{AE}$, posteriormente maximizados em função da aeração. As curvas de $\mathrm{AE}$ ótimas referentes à cada aeração utilizada foram ajustadas polinomialmente, para se estabelecer a tendência do comportamento do processo de produção do biossurfactante e determinar a condição ótima de operação.

\section{RESULTADOS E DISCUSSÃO}

É possível observar no decorrer da fermentação que os comportamentos de atividade emulsificante para as condições de aeração estudadas foram diferentes. Este comportamento foi verificado independente da fonte adicional de carbono utilizada, mostrando que a produção de biossurfactante foi influenciada pela taxa de ar fornecida ao meio de fermentação nas diferentes condições nutricionais.

De acordo com os valores encontrados, os experimentos sem fonte adicional de carbono foram os que apresentaram os melhores resultados. Na Figura 2 são mostrados os perfis de AE encontrados para as diferentes aerações utilizadas nos experimentos sem fonte adicional de carbono.

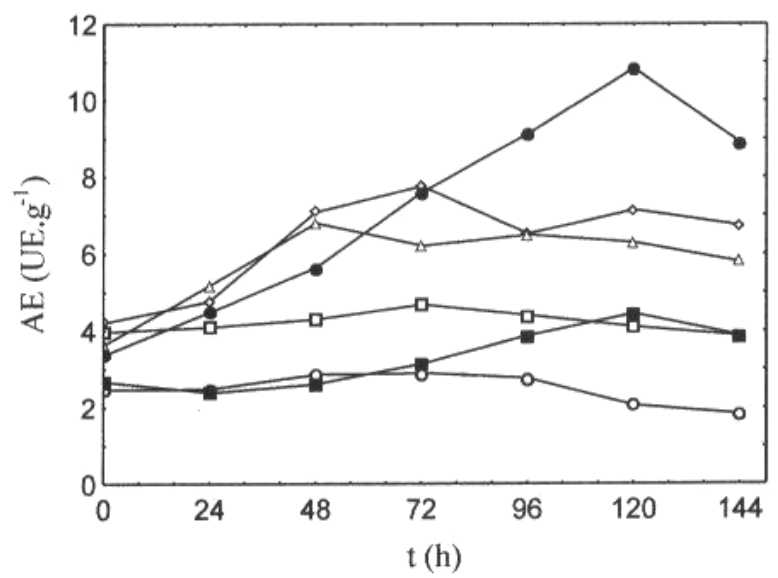

Figura 2. Perfil de atividade emulsificante (AE) em função do tempo de fermentação $(t)$ para os experimentos utilizando $(0) 0,(\square) 40,(\diamond) 60,(\triangle)$ 100, (•) 120 e (অ) $200 \mathrm{~mL}_{\text {ar }} g_{\text {meio }}{ }^{-1} \mathrm{~h}^{-1}$ sem a utilização de fonte adicional de carbono

Veenanadig et al. ${ }^{17}$ estudaram o efeito do fornecimento de ar ao meio de fermentação semi-sólida em biorreatores de coluna com leito fixo utilizando Bacillus subtilis FE-2. Encontraram melhores resultados nos experimentos com maiores taxas de ar. Isto resultou em uma fermentação mais eficiente, observada pelo aumento da quantidade de biossurfactante produzido. No entanto, neste tipo de biorreator, taxas de ar muito elevadas podem ocasionar a diminuição da umidade do meio de fermentação. Tal comportamento foi verificado neste 
trabalho, sendo que o fornecimento de $200 \mathrm{~mL}_{\text {ar }} \mathrm{g}_{\text {meio }}{ }^{-1} \mathrm{~h}^{-1}$ resultou na perda de até $16,02 \%$ da umidade do meio e a conseqüente redução da produção de biossurfactante. A maioria dos experimentos apresentou os melhores resultados de AE em $120 \mathrm{~h}$ de fermentação.

Para verificar se as condições estabelecidas influenciaram no processo de fermentação, foi construído o gráfico de Andrews (Figura 3), seguido da análise de variância dos resultados. Os experimentos frente às diferentes condições de aerações apresentaram diferenças significativas $(p<0,01)$, uma vez que as linhas (que representam cada condição experimental) não se sobrepuseram. Este comportamento também foi verificado nos experimentos utilizando óleo de soja e óleo diesel como fonte adicional de carbono.

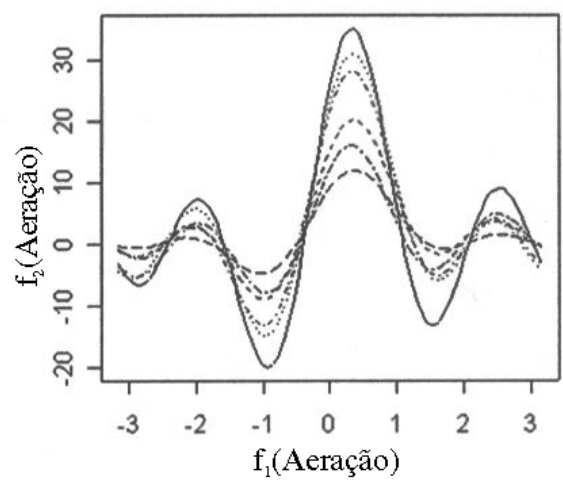

Figura 3. Gráfico de Andrews para os experimentos sem fonte adicional de carbono utilizando diferentes aerações

$\mathrm{Na}$ análise dos resultados algumas condições experimentais apresentaram comportamentos semelhantes, o que sugere a existência de grupos de experimentos com comportamentos parecidos, capaz de representar a influência de algumas variáveis do processo. Para verificar esta afirmação foi aplicada a análise de cluster, mostrada na Figura 4.

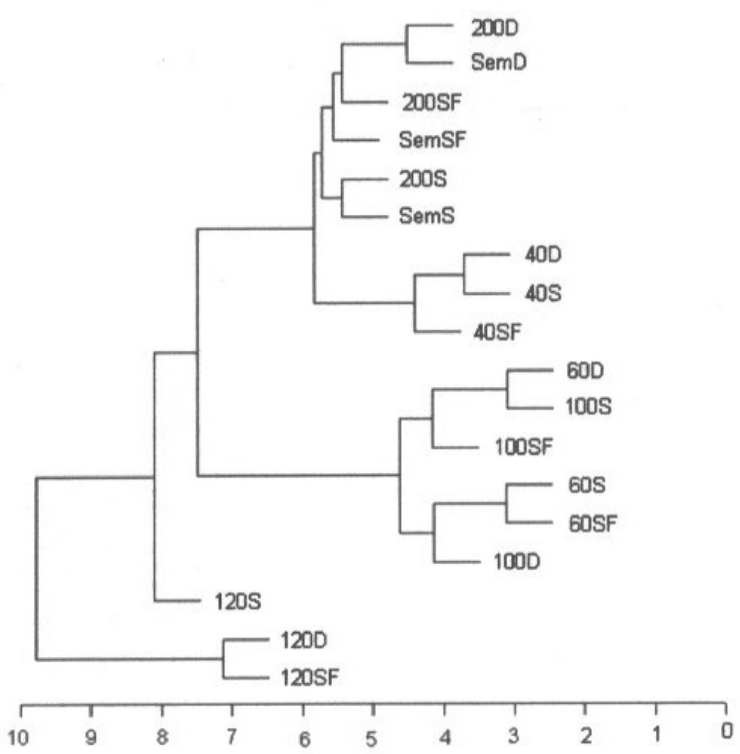

Figura 4. Diagrama de cluster para os resultados de atividade emulsificante, onde as letras referem-se aos experimentos sem fonte adicional de carbono $(S F)$, óleo de soja $(S)$ e óleo diesel $(D)$ e os números correspondem à vazão de $\operatorname{ar}\left(m L_{\text {ar }} g_{\text {meio }}{ }^{-1} h^{-1}\right) e$ "sem" aos experimentos sem fornecimento de ar

Como a análise de cluster é um processo de partição de uma população heterogênea em vários subgrupos mais homogêneos, no agrupamento não há classes pré-definidas, os elementos são agrupados de acordo com a semelhança. Com a aplicação desta técnica de análise classificatória multivariada foi possível verificar a existência de 4 grupos de experimentos com diferentes similaridades em relação à taxa de ar fornecida ao meio de fermentação: Grupo 1 , experimentos sem aeração e com $200 \mathrm{~mL}_{\text {ar }} \mathrm{g}_{\text {meio }}{ }^{-1} \mathrm{~h}^{-1}$; Grupo 2, 60 e $100 \mathrm{~mL}_{\text {ar }} \mathrm{g}_{\text {meio }}{ }^{-1} \mathrm{~h}^{-1}$; Grupo 3, $40 \mathrm{~mL}_{\text {ar }} \mathrm{g}_{\text {meio }}{ }^{-1} \mathrm{~h}^{-1} \mathrm{e}$, Grupo 4, 120 $\mathrm{mL}_{\text {ar }} \mathrm{g}_{\text {meio }}{ }^{-1} \mathrm{~h}^{-1}$. No Grupo 1, o comportamento observado deve-se aos extremos de aeração utilizados, compreendendo o grupo que apresenta os menores valores de AE seguido, em ordem crescente, pelos Grupos 3, 2 e 4.

Os experimentos utilizando $120 \mathrm{~mL}_{\text {ar }} \mathrm{g}_{\text {meio }}{ }^{-1} \mathrm{~h}^{-1}$, por não ter apresentado comportamentos similares, foi considerado como um único grupo (Grupo 4), mostrando que além de apresentar as maiores AE, a influência da fonte adicional de carbono pode ser observada nos melhores resultados encontrados.

O fornecimento de ar ao meio de fermentação apresenta uma importância fundamental para produção de energia a partir da oxidação biológica do carbono presente nos resíduos orgânicos. Parte dessa energia é utilizada no metabolismo dos microrganismos e o restante é liberado na forma de calor. A variação da vazão de ar ao meio de cultivo com Aspergillus fumigatus apresentou uma variação significativa na produção de biossurfactante, o que mostra ser um parâmetro importante para estabelecer as melhores condições do processo. Com isto, a melhor AE proveniente de cada condição de aeração foi ajustada a uma equação polinomial, com objetivo de estabelecer a tendência do comportamento experimental para cada meio de cultivo (Figura 5).

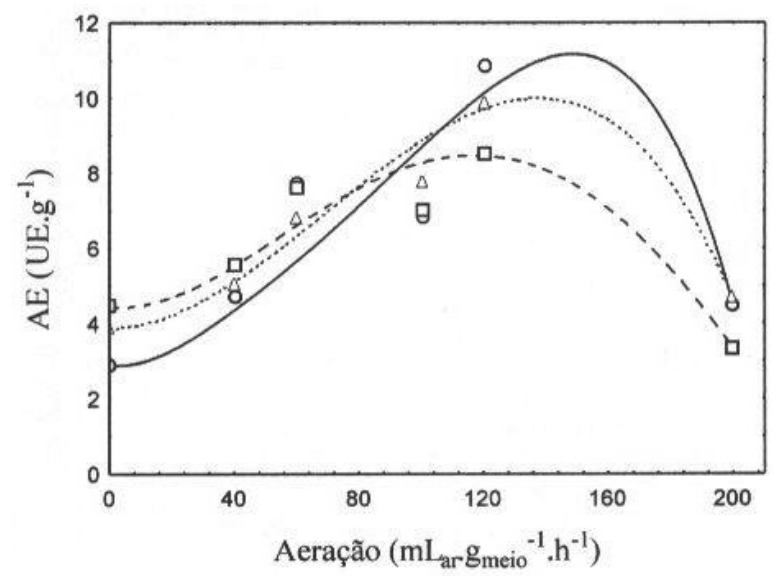

Figura 5. Máximas atividades emulsificantes (AE) encontradas para as diferentes vazões de ar estudadas para os meios (o) sem fonte adicional de carbono, $(\square)$ com óleo de soja e $(\triangle)$ óleo diesel, para seus respectivos resultados ajustados (-), (----) e (....)

Através do ajuste matemático foram gerados 350 pontos referentes a cada condição nutricional utilizada, tornando possível propor a melhor atividade emulsificante para cada uma destas condições. $\mathrm{Na}$ ausência de fonte adicional de carbono foi encontrado 11,17 $\mathrm{UE} \mathrm{g}^{-1}$ de atividade, fornecendo de $148 \mathrm{~mL}_{\text {ar }} \mathrm{g}_{\text {meio }}{ }^{-1} \mathrm{~h}^{-1}$, para os experimentos com óleo diesel 9,99 UE g-1 utilizando $140 \mathrm{~mL}_{\text {ar }} \mathrm{g}_{\text {meio }}{ }^{-1} \mathrm{~h}^{-1}$ e com óleo de soja 8,47 $\mathrm{UE} \mathrm{g}^{-1}$ em $119 \mathrm{~mL}_{\mathrm{ar}} \mathrm{g}_{\text {meio }}{ }^{-1} \mathrm{~h}^{-1}$. Através da diferença encontrada entre os resultados de $\mathrm{AE}$ foi observada a influência do meio nutricional na produção de biossurfactante, mostrando que a ausência de fonte adicional de carbono pode ser vantajosa em relação às demais fontes utilizadas neste processo.

Hommel e Huse ${ }^{18}$ mencionam que os biossurfactantes são predominantemente sintetizados por microrganismos capazes de degradar hidrocarbonetos, mas também podem ser produzidos por 
microrganismos que utilizam como fonte de carbono, glicose, sacarose, glicerol, etanol, entre outros. Vários são os estudos envolvendo a utilização de diferentes substratos na produção de biossurfactantes, mostrando que podem atuar como indutores, influenciando no tipo e no rendimento do biossurfactante. ${ }^{19}$

Os resultados encontrados neste trabalho podem ser explicados pela afirmação de Levišauskas et al. ${ }^{20}$ que mencionam que a concentração de carbono, nitrogênio e fontes de nutrientes específicos para taxa de crescimento celular são os principais fatores que influenciam a biossíntese de biossurfactante. Uma vez que a produção de biossurfactante é dependente do tipo, da quantidade e qualidade da fonte de carbono, ${ }^{21}$ da concentração de $\mathrm{N}, \mathrm{P}, \mathrm{Mg}, \mathrm{Fe}$ e $\mathrm{Mn}$ no meio ${ }^{22,23}$ e das condições físico-químicas, incluindo $\mathrm{pH}$, temperatura, agitação e razão da diluição, ${ }^{24}$ os resultados encontrados sem o uso de fonte adicional de carbono nas condições utilizadas para síntese destes compostos por Aspergillus fumigatos foram as mais vantajosas do ponto de vista prático e econômico.

\section{CONCLUSÃO}

Os experimentos que utilizaram diferentes fontes adicionais de carbono apresentaram comportamentos diferenciados nos resultados de atividade emulsificante para as aerações avaliadas, sendo possível verificar a existência de 4 grupos de experimentos relacionados com o comportamento da produção de biossurfactante: Grupo 1, experimentos sem aeração e com $200 \mathrm{~mL}_{\mathrm{ar}} \mathrm{g}_{\text {meio }}{ }^{-1}$ $\mathrm{h}^{-1}$; Grupo 2, 60 e $100 \mathrm{~mL}_{\text {ar }} \mathrm{g}_{\text {meio }}{ }^{-1} \mathrm{~h}^{-1}$; Grupo 3, $40 \mathrm{~mL}_{\text {ar }} \mathrm{g}_{\text {meio }}{ }^{-1} \mathrm{~h}^{-1} \mathrm{e}$, Grupo 4, $120 \mathrm{~mL}_{\mathrm{ar}} \mathrm{g}_{\text {meio }}{ }^{-1} \mathrm{~h}^{-1}$. A maioria dos experimentos apresentou melhores resultados em $120 \mathrm{~h}$ após o início do processo. As atividades emulsificantes encontradas após o ajuste polinomial dos resultados foram 11,17 $\mathrm{UE} \mathrm{g}^{-1}$ para o meio sem fonte adicional de carbono e aeração $148 \mathrm{~mL} \mathrm{~g}_{\text {meio }}{ }^{-1} \mathrm{~h}^{-1}, 9,99 \mathrm{UE} \mathrm{g}^{-1}$ utilizando óleo diesel e $140 \mathrm{~mL}_{\text {ar }} \mathrm{g}_{\text {meio }}{ }^{-1} \mathrm{~h}^{-1}$ e 8,47 UE g-1 com óleo de soja e $119 \mathrm{~mL}_{\mathrm{ar}}$ $\mathrm{g}_{\text {meio }}{ }^{-1} \mathrm{~h}^{-1}$. O significativo aumento da produção de biossurfactante no meio de cultivo sem fonte adicional de carbono demonstrou ser uma das alternativas mais práticas e economicamente viáveis para a síntese destes compostos em fermentação semi-sólida utilizando Aspergillus fumigatus.

\section{REFERÊNCIAS}

1. Pandey, A.; Selvakumar, P.; Soccol, C. R.; Nigam, P.; Curr. Sci. 1999, 77,149

2. Hesseltine, C. W.; Biotechnol. Bioeng. 1972, 14, 517.

3. Borzani, W.; Aquarone, E.; Lima, U. A.; Schmidell, W.; Biotecnologia Industrial: Engenharia Bioquímica, Edgard Blücher Ltda: São Paulo, 2001, vol. 2

4. Makkar, R. S.; Cameotra, S. S.; Appl. Microbiol. Biotechnol. 2002, 58, 428.

5. Mulligan, C. N.; Yong, R. N.; Gibbs, B. F.; Eng. Geol. 2001, 60, 371.

6. Fiechter A.; Trends Food Sci. Technol. 1992, 31, 283.

7. Crapez, M. A. C.; Borges, A. L. N.; Bispo, M. G.; Pereira, D.; Ciência Hoje 2002, 30, 179.

8. Shafi, R.; Khanna, S.; Indian J. Microbiol. 1995, 33, 163.

9. Rodrigues, L.; Teixeira, J.; Oliveira, R.; Mei, H. C. V.; Process Biochem. 2006, 41, 1 .

10. Makkar, R. S.; Cameotra, S. S.; Surfactants Deterg. 1999, 2, 237.

11. Greek, B. F.; Chem Eng News 1990, 68, 37.

12. Sarney, D. B.; Vulfson, E. N.; Trends Biotechnol. 1995, 13, 164.

13. Layman, P. L.; Chem. Eng. News 1985, 63, 23.

14. Hasan, S. D. M.; Costa, J. A. V.; Sanzo, A. V. L.; Biotech. Tech. 1998, 12,787 .

15. Official Methods of Analysis of the AOAC, $16^{\text {th }}$ ed., Arlington: USA, 1995.

16. Johnson, V.; Singh, M.; Saini, V. S.; Adhikari, D. K.; Sista, V.; Yadav, N. K.; Biotechnol. Lett. 1992, 14, 487.

17. Veenanadig, N. K.; Gowthaman, M. K.; Karanth, N. G. K.; Bioprocess Eng. 2000, 22, 95.

18. Hommel, R. K.; Huse, K.; Biotechnol. Lett. 1993, 15, 853.

19. Makkar, R. S.; Cameotra, S. S.; Surfactants Deterg. 1998, $20,48$.

20. Levišauskas, D.; Galvanauskas, V.; Žunda, G.; Grigiškis, S.; Biotechnol. Lett. 2004, 26, 1141.

21. Georgiou, G.; Lin, S.; Sharma, M. M.; Bioresour. Technol. 1992, 10, 60.

22. Cooper, D. G.; Macdonald, C. R.; Duff, J. B.; Kosaric, N.; Appl. Environ. Microbiol. 1981a, 42, 408.

23. Cooper, D. G.; Zajic, J. E.; Denis, C.; Am. Oil Chem. Soc. 1981b, $58,77$.

24. Lin, S. C.; Carswell, K. S.; Sharma, M. M.; Georgiou, G.; Appl. Microbiol. Biotechnol. 1994, 41, 281. 